\title{
A New Method of Evaluation of Howland Integrals
}

\author{
By Chih-Bing Ling and Jung Lin
}

\begin{abstract}
In this paper, two Howland integrals are evaluated to $25 \mathrm{D}$ when the index is an odd integer. Those Howland integrals when the index is an even integer have been evaluated to $18 \mathrm{D}$ by Nelson. A new method of evaluation is used.
\end{abstract}

The four Howland integrals were first evaluated to 5D by Howland himself, partly with Stevenson, in the papers dealing with a perforated strip [1], [2]. Ling and Nelson in an earlier paper [3] evaluated these integrals to 6D by using a different method through some intermediate integrals. Later, Ling [4] reproduced the 6D values and also added values of a group of related integrals. Recently, Nelson [5], by using the same method, evaluated the integrals to 9D. In the process of computing some related integrals arising from axisymmetrical problems, Nelson, in the same paper, further evaluated the following two Howland integrals to $18 \mathrm{D}$, when $k$ is an even integer:

$$
\begin{array}{ll}
I_{k} & =\frac{1}{2(k !)} \int_{0}^{\infty} \frac{w^{k} d w}{\sinh w \pm w}, \quad(k \geqq 1), \\
\left.I_{k}^{*} w \geqq 3\right) .
\end{array}
$$

The aim of the present paper is to evaluate these two integrals to $25 \mathrm{D}$, when $k$ is an odd integer, by using a direct method without recourse to the intermediate integrals. We begin by expanding the integrands into series as follows:

$$
\begin{aligned}
\frac{w^{k}}{\sinh w \pm w} & =\frac{2 w^{k} e^{-w}}{1 \pm 2 w e^{-w}-e^{-2 w}} \\
& =2 w^{k} e^{-w} \sum_{n=0}^{\infty}(\mp 1)^{n} p_{n}(w) e^{-n w}
\end{aligned}
$$

where $p_{n}(w)$ is the Gegenbauer polynomial of degree $n$ and order unity [6]. The expressions are found to be different depending on $n$ being an even or an odd integer. They are, for $n \geqq 0$,

$$
\begin{aligned}
p_{2 n}(w) & =\sum_{m=0}^{n}(-1)^{n+m}\left(\begin{array}{c}
n+m \\
2 m
\end{array}\right)(2 w)^{2 m}, \\
p_{2 n+1}(w) & =\sum_{m=0}^{n}(-1)^{n+m}\left(\begin{array}{c}
n+m+1 \\
2 m+1
\end{array}\right)(2 w)^{2 m+1} .
\end{aligned}
$$

With the aid of the integral

$$
\int_{0}^{\infty} w^{m} e^{-a w} d w=\frac{m !}{a^{m+1}}, \quad(a>0)
$$

Received October 27, 1969, revised April 21, 1970.

AMS 1969 subject classifications. Primary 6525, 6505.

Key words and phrases. Evaluation of Howland integrals.

Copyright $\odot$ 1971, American Mathematical Society 
the following series are obtained for any integral value of $k$ under the restrictions as indicated,

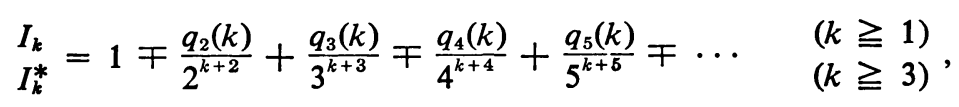

where

$$
\begin{aligned}
& q_{2}(k)=2(k+1), \\
& q_{3}(k)=4 k^{2}+12 k-1, \\
& q_{4}(k)=8(k+1)\left(k^{2}+5 k-2\right), \\
& q_{5}(k)=16 k^{4}+160 k^{3}+260 k^{2}-100 k+409 .
\end{aligned}
$$

In general, $q_{n}(k)$ is a polynomial of $k$ of degree $n-1$ with integral coefficients. The general expressions are

$$
\begin{aligned}
& q_{2 n+1}(k)=\sum_{m=0}^{n}(-1)^{n+m}\left(\begin{array}{c}
2 m+k \\
k
\end{array}\right) \frac{(n+m) !}{(n-m) !} 2^{2 m}(2 n+1)^{2 n-2 m}, \\
& q_{2 n+2}(k)=\sum_{m=0}^{n}(-1)^{n+m}\left(\begin{array}{c}
2 m+k+1 \\
k
\end{array}\right) \frac{(n+m+1) !}{(n-m) !} 2^{2 m+1}(2 n+2)^{2 n-2 m} .
\end{aligned}
$$

The preceding series for the integrals are rapidly convergent when $k$ is a large integer but slowly convergent when $k$ is a small integer. In particular, the series for $I_{1}$ and $I_{2}$ are believed to be only conditionally convergent. For instance, an accuracy of $25 \mathrm{D}$ can be attained with only the first five terms for $k \geqq 44$, ten terms for $k \geqq 33$ and as many as fifty terms for $k \geqq 20$. Hence, it is necessary to use some other method to evaluate the early integrals. By a combined use of Cauchy's integral theorem and Cauchy's theorem of residues, the integrals $I_{2 k-1}$ and $I_{2 k-1}^{*}$ are developed into series as follows: For $k \geqq 1$,

$$
\begin{aligned}
& I_{2 k-1}=\frac{1}{2(2 k-1) !}\left\{\frac{\alpha}{4} \delta_{k, 1}+\alpha \sum_{n=1}^{\infty} \frac{(n \alpha)^{2 k-1}}{\sinh n \alpha+n \alpha}\right.\left.+\operatorname{Re} \sum_{m=1}^{\infty} \frac{\pi \pi_{m}^{2 k-1} \exp \left(\pi i z_{m} / \alpha\right)}{\cosh ^{2}\left(z_{m} / 2\right) \sin \left(\pi z_{m} / \alpha\right)}\right\} \\
& I_{2 k-1}=\frac{1}{2(2 k-1) !}\left\{\alpha \sum_{n=0}^{\infty} \frac{\left(n \alpha+\frac{1}{2} \alpha\right)^{2 k-1}}{\sinh \left(n+\frac{1}{2}\right) \alpha+\left(n+\frac{1}{2}\right) \alpha}\right. \\
&\left.+\operatorname{Re} \sum_{m=1}^{\infty} \frac{\pi i z_{m}^{2 k-1} \exp \left(\pi i z_{m} / \alpha\right)}{\cosh ^{2}\left(z_{m} / 2\right) \cos \left(\pi z_{m} / \alpha\right)}\right\}
\end{aligned}
$$

and for $k \geqq 2$,

$$
\begin{aligned}
& I_{2 k-1}^{*}=\frac{1}{2(2 k-1) !}\left\{3 \alpha \delta_{k, 2}+\alpha \sum_{n=1}^{\infty} \frac{(n \alpha)^{2 k-1}}{\sinh n \alpha-n \alpha}\right.\left.+\operatorname{Re} \sum_{m=1}^{\infty} \frac{\pi\left(z_{m}^{*}\right)^{2 k-1} \exp \left(\pi i z_{m}^{*} / \alpha\right)}{\sinh ^{2}\left(z_{m}^{*} / 2\right) \sin \left(\pi z_{m}^{*} / \alpha\right)}\right\} \\
& I_{2 k-1}^{*}=\frac{1}{2(2 k-1) !}\left\{\alpha \sum_{n=0}^{\infty} \frac{\left(n \alpha+\frac{1}{2} \alpha\right)^{2 k-1}}{\sinh \left(n+\frac{1}{2}\right) \alpha-\left(n+\frac{1}{2}\right) \alpha}\right. \\
&\left.+\operatorname{Re} \sum_{m=1}^{\infty} \frac{\pi i\left(z_{m}^{*}\right)^{2 k-1} \exp \left(\pi i z_{m}^{*} / \alpha\right)}{\sinh ^{2}\left(z_{m}^{*} / 2\right) \cos \left(\pi z_{m}^{*} / \alpha\right)}\right\},
\end{aligned}
$$


where $\alpha$ is a positive constant, $\delta_{k, n}$ is the Kronecker delta, and $z_{m}$ and $z_{m}^{*}$ are the $m$ th complex zeros of $\sinh z \pm z$, respectively, in the first quadrant of the $z$-plane. The results give two different expressions for each integral. The derivation will be described later. Each integral can then be computed from one expression and checked by the other.

It is seen that each expression consists of two series. The first one is a real series and the second the real part of a complex series. It is also seen that the constant $\alpha$ occurs only on the right-hand side of each expression. This constant can be fixed to suit our convenience. The first series converges rapidly when $\alpha$ is large and the second when $\alpha$ is small. In fact, the first series of the first expression of each integral represents the value given by the trapezoidal rule, and the first series of the second expression represents the value given by the rectangular rule. In both cases, $\alpha$ is the width of the strip. Therefore, the second series of each expression may be regarded merely as a correction, analogous to the second series in Gregory's formula [7]. By a proper choice of $\alpha$, the value of the second series can generally be made small in comparison with that of the first series.

In the computation, the value of $\alpha$ is taken as unity. Unlike the series in (5), the convergence of the first series becomes slower as $k$ increases. To attain an accuracy of $25 \mathrm{D}$ with this value of $\alpha, 65$ terms of the first series are needed for $k=1,130$ terms for $k=15$ and 200 terms for $k=35$. The corresponding numbers of terms needed to attain an accuracy of 18D are 47, 110 and 175, respectively. The number of terms needed in each instance decreases to one half if the value of $\alpha$ is doubled. In computing the second series, the $11 \mathrm{D}$ values of $z_{m}$ and $z_{m}^{*}$ computed before by Ling [8] are available. Their accuracy can be improved readily, whenever needed, by using the Newton-Raphson method. The convergence of the second series is so rapid that when $\alpha=1$, at most two terms are needed for the present computation.

An alternative method for computing these values is to attempt to evaluate the remainder term in (5). This remainder term has an integral representation of a similar nature as $I_{k}$ or $I_{k}^{*}$ itself, but the integrand is more complicated. On the other hand, when the Gauss-Laguerre quadrature rule was used in the evaluation, it was found that, for small values of $k$, adequate precision could not be obtained without an effort far exceeding that required when using (8) and (9).

The computation was carried out on an IBM 1620 computer. The following relations were used as a further check:

$$
\begin{aligned}
& \sum_{k=1}^{\infty}\left(1-I_{2 k-1}\right)=I_{1}-\frac{1}{4}, \\
& \sum_{k=2}^{\infty}\left(I_{2 k-1}^{*}-1\right)=\frac{5}{4} .
\end{aligned}
$$

Some typical values of $I_{k}$ and $I_{k}^{*}$ rounded to 25D are shown in the accompanying table. The complete results for odd integral values of $k$ up to 91 appear in the tables on pp. 334 and 335 of this paper.

Derivation of Expressions (8) and (9). Consider the contour integral

$$
\frac{1}{2 \pi i} \oint \frac{z^{2 k-1} d z}{(z-t)(\sinh z+z) \cos (\pi z / \alpha)}, \quad(k \geqq 1),
$$


Table. Howland integrals $I_{k}$ and $I_{k}^{*}$ when $k$ is an odd integer

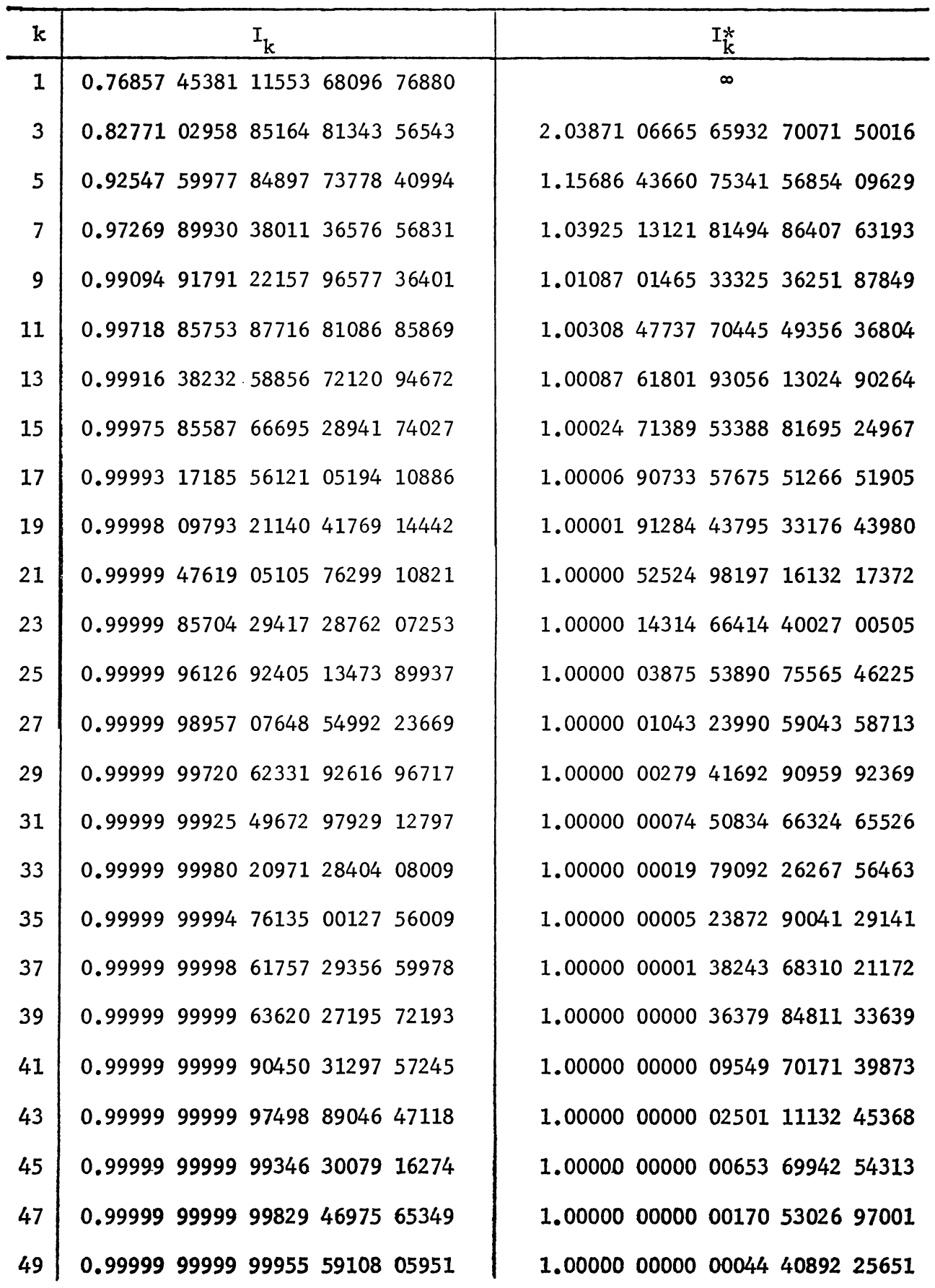


Table. Howland integrals $I_{k}$ and $I_{k}^{*}$ when $k$ is an odd integer (cont'd)

\begin{tabular}{|c|c|c|c|c|c|c|c|c|c|}
\hline k & & $\mathrm{I}_{\mathrm{k}}$ & & & \multicolumn{5}{|c|}{$I_{k}^{*}$} \\
\hline 51 & 0.9999999999 & 99988 & 45368 & 07336 & 1.00000 & 00000 & 00011 & 54631 & 96458 \\
\hline 53 & 0.9999999999 & 99997 & 00239 & 78562 & 1.00000 & 00000 & 00002 & 99760 & 21892 \\
\hline 55 & 0.9999999999 & 99999 & 22284 & 38855 & 1.00000 & 00000 & 00000 & 77715 & 61199 \\
\hline 57 & 0.9999999999 & 99999 & 79877 & 20771 & 1.00000 & 00000 & 00000 & 20122 & 79235 \\
\hline 59 & 0.9999999999 & 99999 & 94795 & 82958 & 1.00000 & 00000 & 00000 & 05204 & 17043 \\
\hline 61 & 0.9999999999 & 99999 & 98655 & 58931 & 1.00000 & 00000 & 00000 & 01344 & 41069 \\
\hline 63 & 0.9999999999 & 99999 & 99653 & 05530 & 1.00000 & 00000 & 00000 & 00346 & 94470 \\
\hline 65 & 0.9999999999 & 99999 & 99910 & 55332 & 1.00000 & 00000 & 00000 & 00089 & 44668 \\
\hline 67 & 0.9999999999 & 99999 & 99976 & 96070 & 1.00000 & 00000 & 00000 & 00023 & 03930 \\
\hline 69 & 0.9999999999 & 99999 & 99994 & 07077 & 1.00000 & 00000 & 00000 & 00005 & 92923 \\
\hline 71 & 0.9999999999 & 99999 & 99998 & 47534 & 1.00000 & 00000 & 00000 & 00001 & 52466 \\
\hline 73 & 0.9999999999 & 99999 & 99999 & 60825 & 1.00000 & 00000 & 00000 & 00000 & 39175 \\
\hline 75 & 0.9999999999 & 99999 & 99999 & 89941 & 1.00000 & 00000 & 00000 & 00000 & 10059 \\
\hline 77 & 0.9999999999 & 99999 & 99999 & 97419 & 1.00000 & 00000 & 00000 & 00000 & 02581 \\
\hline 79 & 0.9999999999 & 99999 & 99999 & 99338 & 1.00000 & 00000 & 00000 & 00000 & 00662 \\
\hline 81 & 0.9999999999 & 99999 & 99999 & 99830 & 1.00000 & 00000 & 00000 & 00000 & 00170 \\
\hline 83 & 0.9999999999 & 99999 & 99999 & 99957 & 1.00000 & 00000 & 00000 & 00000 & 00043 \\
\hline 85 & 0.9999999999 & 99999 & 99999 & 99989 & 1.00000 & 00000 & 00000 & 00000 & 00011 \\
\hline 87 & 0.9999999999 & 99999 & 99999 & 99997 & 1.00000 & 00000 & 00000 & 00000 & 00003 \\
\hline 89 & 0.9999999999 & 99999 & 99999 & 99999 & 1.00000 & 00000 & 00000 & 00000 & 00001 \\
\hline 91 & 1.0000000000 & 00000 & 00000 & 00000 & 1.00000 & 00000 & 00000 & 00000 & 00000 \\
\hline
\end{tabular}


TABLE

\begin{tabular}{|c|c|c|c|c|c|c|c|c|c|}
\hline$k$ & \multicolumn{4}{|c|}{$I_{k}$} & \multicolumn{5}{|c|}{$I_{k}^{*}$} \\
\hline 1 & $0.76857 \quad 45381$ & 11553 & 68096 & 76880 & & & $\infty$ & & \\
\hline 3 & $\begin{array}{lll}0.82771 & 02958\end{array}$ & 85164 & 81343 & 565 & 2.03 & 06665 & 65932 & 70071 & 50016 \\
\hline 5 & 759977 & 84897 & 73778 & 40994 & & 43660 & 75341 & 56854 & 09629 \\
\hline 15 & 5855 & 66695 & 28941 & 740 & 24 & 71389 & 53388 & 81695 & 24 \\
\hline 25 & 0.9999996126 & 92405 & 13473 & 89937 & 1.0 & 03875 & 53890 & 75565 & 46225 \\
\hline 35 & 0.9999999994 & 76135 & 00127 & 56009 & 1.00000 & 00005 & 23872 & 90041 & 29141 \\
\hline 55 & 0.9999999999 & 99999 & 22284 & 38855 & & 00000 & 00000 & 77715 & 61199 \\
\hline 85 & 0.9999999999 & 99999 & 99999 & 99989 & 1.00000 & 00000 & 00000 & 00000 & 00011 \\
\hline
\end{tabular}

where the contour is taken round the circle $|z|=R$ through a sequence of values such that the circle never passes through any pole of the integrand, $t$ being any point on the $x$ axis inside the circle and $\alpha$ a positive constant. The integral tends to zero as $R$ tends to infinity. The poles of the integrand are

$$
z=t, \quad z= \pm z_{m}, \quad z= \pm \bar{z}_{m}, \quad z= \pm\left(n+\frac{1}{2}\right) \alpha
$$

where $m=1,2,3, \cdots, n=0,1,2, \cdots$, and a bar denotes the complex conjugate, $z_{m}$ being defined before. Note that the complex zeros of $\sinh z+z$ in the entire $z$-plane are symmetrically located in each quadrant with respect to both the $x$ and $y$ axes. Furthermore, the origin $z=0$ is also a zero. Both poles are of order unity.

It follows from Cauchy's theorems that the sum of residues at all the poles is zero. Consequently, we find

$$
\begin{aligned}
\frac{t^{2 k-1}}{(\sinh t+t) \cos (\pi t / \alpha)}= & \frac{\alpha}{\pi} \sum_{n=0}^{\infty} \frac{2(-1)^{n}\left(n \alpha+\frac{1}{2} \alpha\right)^{2 k}}{\sinh \left(n+\frac{1}{2}\right) \alpha+\left(n+\frac{1}{2}\right) \alpha} \cdot \frac{1}{\left(n \alpha+\frac{1}{2} \alpha\right)^{2}-t^{2}} \\
& -\operatorname{Re} \sum_{m=1}^{\infty} \frac{2 z_{m}^{2 k}}{\cosh ^{2}\left(z_{m} / 2\right) \cos \left(\pi z_{m} / \alpha\right)} \cdot \frac{1}{z_{m}^{2}-t^{2}} .
\end{aligned}
$$

Multiplying by $\cos (\pi t / \alpha)$, integrating with respect to $t$ from zero to infinity, and making use of the following integrals

$$
\begin{aligned}
\int_{0}^{\infty} \frac{\cos (\pi t / \alpha) d t}{\left(n \alpha+\frac{1}{2} \alpha\right)^{2}-t^{2}} & =\frac{(-1)^{n} \pi}{(2 n+1) \alpha}, \\
\int_{0}^{\infty} \frac{\cos (\pi t / \alpha) d t}{z_{m}^{2}-t^{2}} & =-\frac{\pi i}{2 z_{m}} \exp \left(\pi i z_{m} / \alpha\right),
\end{aligned}
$$

we find the second expression in (8).

Again, consider the contour integral

$$
\frac{1}{2 \pi i} \oint \frac{z^{2 k-1} d z}{(z-t)(\sinh z+z) \sin (\pi z / \alpha)}, \quad(k \geqq 1) .
$$

The poles of the integrand are

$$
z=t, \quad z= \pm z_{m}, \quad z= \pm \bar{z}_{m}, \quad z= \pm n \alpha,
$$


where $m=1,2,3, \cdots$ and $n=1,2,3, \cdots$. In particular, when $k=1$, an additional pole is at the origin $z=0$. By making use of the following integrals

$$
\begin{aligned}
& \int_{0}^{\infty} \frac{t \sin (\pi t / \alpha) d t}{(n \alpha)^{2}-t^{2}}=-\frac{(-1)^{n} \pi}{2}, \\
& \int_{0}^{\infty} \frac{t \sin (\pi t / \alpha) d t}{z_{m}^{2}-t^{2}}=-\frac{\pi}{2} \exp \left(\pi i z_{m} / \alpha\right),
\end{aligned}
$$

we find similarly the first expression in (8).

By replacing $\sinh z+z$ with $\sinh z-z$ in the foregoing two contour integrals, we likewise find the two expressions in (9) for $k \geqq 2$.

It should be mentioned that two expressions for each $I_{2 k}$ and $I_{2 k}^{*}$ can be derived in a similar manner. However, the resulting expressions appear to be less simple since they also involve sine and cosine integrals. It should also be mentioned that the foregoing method of evaluation can be generalized.

Department of Mathematics

Virginia Polytechnic Institute and State University

Blacksburg, Virginia 24061

Department of Physics

Tennessee Technological University

Cookeville, Tennessee 38501

1. R. C. J. Howland, "On the stresses in the neighbourhood of a circular hole in a strip under tension," Philos. Trans. Roy. Soc. London Ser. A, v. 229, 1930, 49-86.

2. R. C. J. Howland \& A. C. STEvenson, "Biharmonic analysis in a perforated strip," Philos. Trans. Roy. Soc. London Ser. A, v. 232, 1934, pp. 155-222.

3. C. B. Ling \& C. W. Nelson, "On evaluation of Howland integrals," Ann. Acad. Sinica, Taiwan, v. 2, part 2, 1955, pp. 45-50.

4. C. B. LING, "Tables of values of 16 integrals of algebraic-hyperbolic type," MTAC, v. 11,1957 , pp. $160-166$. MR 19, 886.

5. C. W. Nelson, "New tables of Howland's and related integrals," Math. Comp., v. 15, 1961, pp. 12-18. MR 22 \#10203.

6. A. ERDÉLYI, ET AL., Higher Transcendental Functions. Vol. I, McGraw-Hill, New York, 1953, pp. 175-176. MR 15, 419.

7. E. T. WhITTAKER \& G. Robinson, Calculus of Observations, 4th ed., Blackie, London, 1948, pp. $143-144$.

8. C. B. LING \& F. H. CheNG, "Stresses in a semi-infinite strip," Internat. J. Engrg. Sci., v. 5,1967, pp. $155-170$. 